\title{
Risk stratification model for patients with stage I invasive lung adenocarcinoma based on clinical and pathological predictors
}

\author{
Yiyang Wang ${ }^{1 \#}$, Difan Zheng ${ }^{2 \#}$, Jizhuang Luo ${ }^{1}$, Jie Zhang $^{3}$, Cecilia Pompili ${ }^{4}$, Hideki Ujiie ${ }^{5}$, \\ Natsumi Matsuura ${ }^{6}$, Haiquan Chen ${ }^{2,7,8}$, Feng Yao ${ }^{1}$
}

${ }^{1}$ Department of Thoracic Surgery, Shanghai Chest Hospital, Shanghai Jiao Tong University, Shanghai, China; ${ }^{2}$ Department of Thoracic Surgery and State Key Laboratory of Genetic Engineering, Fudan University Shanghai Cancer Center, Shanghai, China; ${ }^{3}$ Department of Pathology, Shanghai Chest Hospital, Shanghai Jiao Tong University, Shanghai, China; ${ }^{4}$ Department of Thoracic Surgery, University of Leeds, Section of Patient Centred Outcomes Research, Leeds Institute for Medical Research at St. James's, St. James' Institute of Oncology, Leeds, UK; ${ }^{5}$ Department of Cardiovascular and Thoracic Surgery, Hokkaido University, Hokkaido, Japan; ${ }^{6}$ Department of General Thoracic Surgery, Japanese Red Cross Maebashi Hospital, Asakura, Maebashi City, Gunma, Japan; ${ }^{7}$ Institutes of Thoracic Oncology, Fudan University, Shanghai, China; ${ }^{8}$ Department of Oncology, Shanghai Medical College, Fudan University, Shanghai, China

Contributions: (I) Conception and design: H Chen, F Yao; (II) Administrative support: C Pompili, H Ujiie, N Matsuura; (III) Provision of study materials or patients: Y Wang, D Zheng; (IV) Collection and assembly of data: Y Wang, D Zheng; (V) Data analysis and interpretation: J Luo, J Zhang; (VI) Manuscript writing: All authors; (VII) Final approval of manuscript: All authors.

\#These authors contributed equally to this work.

Correspondence to: Haiquan Chen, MD. Department of Thoracic Surgery, Fudan University Shanghai Cancer Center, 270 Dong-An Road, Shanghai 200032, China. Email: hqchen1@yahoo.com; Feng Yao, MD. Department of Thoracic Surgery, Shanghai Chest Hospital, Shanghai Jiao Tong University, no. 241 West Huaihai Road, Shanghai 200030, China. Email: yaofeng6796678@126.com.

Background: The aim of this study was to propose a new kind of pathological classification and further establish a prognostic model for resected stage I invasive adenocarcinoma (IADC).

Methods: Clinicopathological data were collected from 2 hospitals. The new proposed pathological reclassification was defined according to certain subtype instead of a predominant one. Survival curves were plotted by Kaplan-Meier analysis. Cox regressions were analyzed for recurrence-free survival (RFS) and overall survival (OS), through which prognostic scores and stratification models were established. The comparison between risk models and the eighth edition of tumor, node, metastasis (TNM) classification was conducted through receiver operating characteristic curves (ROC), as identified by the area under the curve (AUC) and $z$ test.

Results: In all, 1,196 patients were enrolled. At multivariable analysis, solid and micropapillary of the new pathological reclassification, along with stage IA3 and IB were independent predictors for poorer RFS. Stage IB and smoking status significantly indicated worse OS. After normalization and standardization of log-hazard ratio (HR), personalized scores were calculated and the risk stratifications with 3 risk groups were generated. Compared with TNM classification, the risk model of RFS showed advantage over earlyrecurrence prediction (1-year: 0.653 vs. $0.556, \mathrm{P}=0.033$; 3 -year: 0.663 vs. $0.076, \mathrm{P}=0.008$ ). No marked difference was observed in long-term RFS or OS.

Conclusions: Considering the harboring of certain patterns may be a new concept in adenocarcinoma classification. The risk stratification model based on this pathological classification and the eighth TNM classification showed remarkable superiority over TNM alone in predicting early recurrence of stage I adenocarcinoma. However, TNM classification remained valuable for long-term recurrence and survival prediction.

Keywords: Pathological stage I lung invasive adenocarcinoma; new pathological classification; risk stratification model; the eighth edition TNM classification 
Submitted Mar 02, 2021. Accepted for publication May 21, 2021.

doi: $10.21037 /$ tlcr-21-393

View this article at: http://dx.doi.org/10.21037/tlcr-21-393

\section{Introduction}

Non-small-cell lung cancers (NSCLC) have become a severe public health issues across all countries of the world, and especially in China, which currently has an all-time high level of NSCLC-related morbidity and mortality $(1,2)$. As one of the major subtypes of NSCLC, lung adenocarcinoma (ADC) accounts for approximately $70 \%$ of NSCLC cases (3). Although lobectomy has been recommended as the first choice for early-staged NSCLC, with sublobar resection as an appropriate substitution to well-selected patients (4-6), the 5-year survival rate still remains dissatisfactory (7).

With the widespread use of low-dose computed tomography (LDCT), there has been a significant boost in the detection of early-stage NSCLC, particularly smallsized ADC (8). The tumor, node, metastasis (TNM) classification system has been considered the gold standard for lung cancer survival outcome for decades; the eighth TNM edition of lung cancer indicates a 5 -year survival of $90 \%, 85 \%, 80 \%$, and $73 \%$ for stage IA1, IA2, IA 3 , and IB cases, respectively (9). However, despite this reliable prognostic survival, the application of the TNM system alone might not be able to indicate the risk of tumor recurrence after complete resection (7).

The International Association for the Study of Lung Cancer (IASLC)/American Thoracic Society (ATS)/ European Respiratory Society (ERS) have recognized the predominant histologic subtype as the standard classification to resected ADC. The subtypes with predominant patterns include lepidic-predominant ADC (LEP), acinar-predominant ADC (ACN), papillarypredominant ADC (PAP), solid-predominant ADC (SOL), micropapillary-predominant ADC (MIP) and other variants $(10,11)$. However, ADC displays the most heterogeneity with the most mixed histological patterns compared with other non-ADC lung cancers, different subtype of ADC with varying potential influences on recurrence and the postsurgical adjuvant therapy $(12,13)$. For instance, the poor prognosis of ADC patients existed even when the SOL/MIP components were not in predominance and postoperative therapy should be carefully considered (14). Besides, invasive adenocarcinoma (IADC) patients harboring LEP component were clearly correlated with relatively better prognosis compared with those without LEP component according to our previous studies $(15,16)$. Hence, we hypothesized that the specific subtype of stage I ADC rather than the predominant type would truly be indicative of the recurrence and survival outcome.

In this research, we tried to establish a risk stratification model combining other independent prognostic factors with the eighth edition TNM classification and extend the content of the TNM classification system. Therefore, we defined a proposed pathological classification with respect to the specific subtype of ADC and further developed a clinicopathological risk stratification model for the personalized prediction of pathological stage I IADC based on the data pool from Shanghai Chest Hospital and Fudan University Shanghai Cancer Center.

We present the following article in accordance with the TRIPOD reporting checklist (available at http://dx.doi. org/10.21037/tlcr-21-393).

\section{Methods}

\section{Patients}

All procedures performed in this study involving human participants were in accordance with the Declaration of Helsinki (as revised in 2013). The study was approved by ethics board of Shanghai Chest Hospital (KS1992) and informed consent was not needed. A total of 3,368 cases with resected pulmonary nodules $\leq 3 \mathrm{~cm}$ admitted from January 2007 to December 2011 to the Shanghai Chest Hospital and Fudan University Shanghai Cancer Center were screened for eligibility for investigation. According to the inclusion and exclusion criteria (Figure 1), 1,196 cases with pathological stage I IADC were enrolled. Non-ADC lesions such as squamous carcinoma, large-cell and smallcell lung cancer; noninvasive ADC including atypical adenomatous hyperplasia (AAH), ADC in situ (AIS), and minimally invasive ADC (MIA) identified by final pathology; multiple lesions indicating multiple primary ADC confirmed by examination and pathology; patients who received neoadjuvant therapy or palliative treatment; the local extension indicating advanced staging such as 


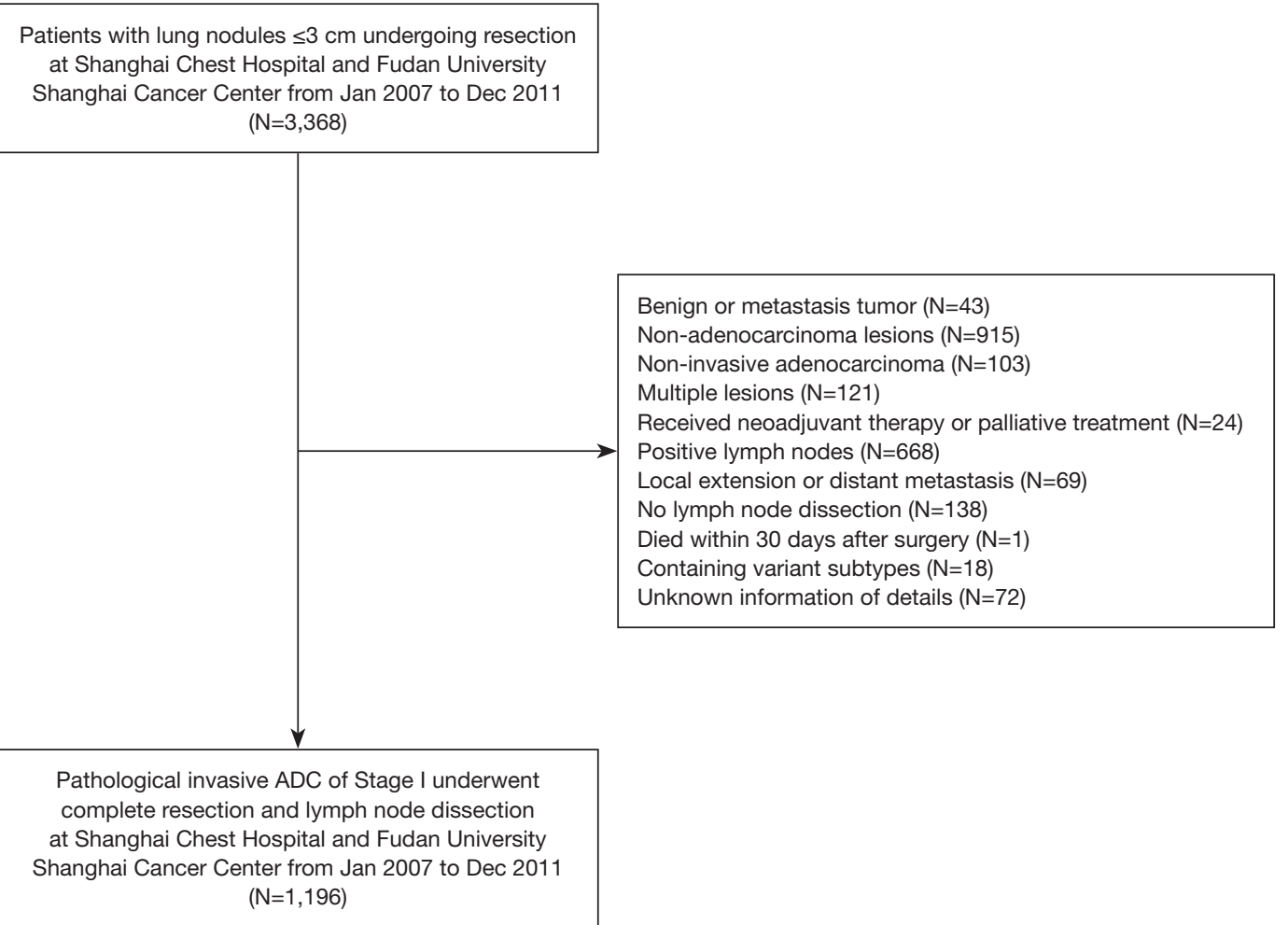

Figure 1 Study cohort flowchart. A total of 3,368 patients with pulmonary nodules $\leq 3 \mathrm{~cm}$ who underwent surgical resection at Shanghai Chest Hospital and Fudan University Shanghai Cancer Center were identified between January 2007 and December 2011. After exclusion, 1,196 patients were included in this study.

$\mathrm{T} 3$ and T4 with respect to the eighth TNM classification; patients who died within 30 days of surgery; cases with positive lymph nodes; and cases with no dissection of lymph nodes in the hilum or mediastinum. In addition, patients with variant subtypes or those with missing data were also excluded.

\section{Outcomes variables and patient follow-up}

The primary endpoint was recurrence-free survival (RFS), defined as survival from the date of surgical operation to the date of recurrence or last follow-up. The secondary endpoint was overall survival (OS), defined as the survival between surgery and death or last follow-up. All patients completed the preoperative routine examinations including enhanced chest computed tomography (CT) scans, head CT scans or magnetic resonance imaging (MRI), abdominal ultrasound, and bone scintigraphy. Positron emission tomography (PET) was performed when required. After the surgery, our routine follow-up time was every 3 months for the first postoperative year; CT scans, neck and abdominal ultrasound, head MRI, and bone scintigraphy were conducted at postoperative month 6 and repeated every year thereafter. Patients would be contacted via telephone calls prior to their follow-up with appointments fixed in the outpatient department.

\section{Definition and identification of the proposed new risk pathological classification of IADC}

In this study, we proposed a new pathological classification system for ADC. To be specific, any subtype of IADC harboring MIP was classified under the new classification of MIP (nMIP). The subtype containing SOL not coexisting with MIP was classified as the new classification of SOL (nSOL). Histological subtypes containing PAP except for MIP/SOL were therefore classified as nPAP. The proposed new classification of nACN was defined as ADC containing ACN instead of MIP/SOL/PAP. The remaining pathological subtypes were divided into nLEP (containing 
LEP without MIP/SOL/PAP/ACN) and others. For a more accurate and unbiased assessment, hematoxylin and eosin (HE)-stained slides of the pathological samples were all rechecked by 2 experienced pathologists from each hospital.

\section{Statistical analysis}

The association between the categorical variables was calculated by Pearson $\chi^{2}$ or Fisher's exact test. Univariable and multivariable Cox regressions were conducted to investigate the potential independent prognostic factors of RFS and OS as estimated by hazard ratio (HR) and $95 \%$ confidence interval ( $95 \% \mathrm{CI})$. The results of the HR obtained above were registered as the status of log-HR, from which the personalized risk scores were generated $(17,18)$. Then, normalization and standardization of these scores were divided by the smallest value of the obtained risk coefficients and the accumulation of personalized scores for patients were derived (18). Thus, the risk score stratification model (including low-, intermediate-, and high-risk classes) for patients' clinical outcomes could be presented and the cutoffs, which were decided upon by the trisection of risk values (low risk class with score $\geq 0$ and $<1$, intermediate risk class with scores $\geq 1$ and $<2$, and high risk class with scores $\geq 2$ ). Kaplan-Meier survival curves were plotted for comparison between risk model and the eighth TNM classification, and identified by the log-rank test. The curves of receiver operating characteristics (ROC) were performed to analyze the sensitivity and specificity between our risk model and the eighth TNM classification through the estimation of the area under the curve (AUC), the difference of which was verified by $z$ test.

\section{Results}

\section{The baseline characteristics of pathological stage I LADC}

The clinicopathological characteristics of 1,196 patients are listed in Table 1. The predominant patterns were classified according to the 2015 World Health Organization classification, and included lepidic-predominant ADC (LEP), acinar-predominant ADC (ACN), papillary-predominant ADC (PAP), solid-predominant ADC (SOL), micropapillarypredominant ADC (MIP), and other variants (7). The proposed pathological classification was classified according to our previous definition. The relationship between the predominant patterns and our proposed classification can been seen in Table S1.

The primary cohort mainly consisted of females (61\%), and more than half $(54 \%)$ of our candidates were aged $\leq 60$ years. With respect to smoking status, the majority (almost $64 \%$ ) of patients were never-smokers, while the smoking patients (including cessation) accounted for less than $36 \%$ of the total. Therefore, the neversmokers constituted the majority of the candidates. As for the location of ADC, the most common site of all the resected stage I IADC patients was still the right upper lobe (RUL), accounting for $32.6 \%$ of cases, followed by the left upper lobe at $25 \%$, the right lower lobe (RLL) at $18.9 \%$, the left lower lobe (LLL) at $14.1 \%$, and the right middle lobe (RML) at $9.4 \%$. In this study, patients without adjuvant chemotherapy were still in the majority, accounting for $76.5 \%$ of patients; meanwhile, patients with adjuvant chemotherapy (including standard adjuvant chemotherapy and incomplete one) constituted only $23.5 \%$ of patients. As for the tumor size of the resected stage I ADC cases, the majority of our primary cohort consisted of tumor sizes of 1 to $2 \mathrm{~cm}$ and 2 to $3 \mathrm{~cm}$, at $47.4 \%$ and $43.2 \%$, respectively. Meanwhile, sizes below $1 \mathrm{~cm}$ accounted for fewer than $9.4 \%$ of cases, probably owing to the lower aggressiveness of smaller-sized tumors. Lobectomy is still recommended as the gold stand approach worldwide. Hence, nearly $96.7 \%$ of patients underwent lobectomy in our primary cohort, while only $3.3 \%$ underwent anatomical sublobar resections. And all the patients received systemic lymph dissection or sampling. For pathological subtypes, ACN and PAP were the two most common subtypes among stage I ADC patients, accounting for $46.7 \%$ and $36 \%$ of cases, respectively. These subtypes were followed by SOL, MIP, and IMA, all of which had lower than $5 \%$ predominance. Specifically, MIP accounted for less than $0.5 \%$ of all subtype, which indicated a potential relationship between the subtype and high invasiveness and metastatic ability. In reference to our proposed new pathological classification, nPAP accounted for the majority of our primary cohort $(54.2 \%)$, followed by the classification of nACN (23.1\%), nSOL (11.4\%), and nLEP (5.3\%). nMIP and others accounted for only $3 \%$, respectively. We selected the eighth TNM classification as our standard classification in this study and classified our resected stage I IADC patients into stage IA1, IA2, IA3, and IB accordingly. The stage IA2 IADC patients accounted for the majority (35.4\%) of patients, followed by stage IB (29.9\%), stage IA3 (26\%), and stage IA1 
Table 1 Baseline characteristics of the primary cohort with stage I invasive adenocarcinoma who underwent complete resection and lymph node dissection

\begin{tabular}{|c|c|c|}
\hline Variables & No. of patients & (\%) \\
\hline \multicolumn{3}{|l|}{ Sex } \\
\hline Male & 468 & 39.1 \\
\hline Female & 728 & 60.9 \\
\hline \multicolumn{3}{|l|}{ Age } \\
\hline$\leq 60$ years & 645 & 53.9 \\
\hline$>60$ years & 551 & 46.1 \\
\hline \multicolumn{3}{|c|}{ Smoking history } \\
\hline No & 764 & 63.9 \\
\hline Yes & 432 & 36.1 \\
\hline \multicolumn{3}{|l|}{ Location } \\
\hline RUL & 390 & 32.6 \\
\hline $\mathrm{RML}$ & 113 & 9.4 \\
\hline RLL & 226 & 18.9 \\
\hline LUL & 299 & 25 \\
\hline LLL & 168 & 14.1 \\
\hline \multicolumn{3}{|c|}{ Adjuvant chemotherapy } \\
\hline No & 915 & 76.5 \\
\hline Yes & 281 & 23.5 \\
\hline \multicolumn{3}{|l|}{ Size } \\
\hline$\leq 1 \mathrm{~cm}$ & 113 & 9.4 \\
\hline$\leq 2 \mathrm{~cm}$ & 566 & 47.4 \\
\hline$\leq 3 \mathrm{~cm}$ & 517 & 43.2 \\
\hline \multicolumn{3}{|l|}{ Surgery } \\
\hline Sublob & 40 & 3.3 \\
\hline Lob & 1,156 & 96.7 \\
\hline \multicolumn{3}{|l|}{ Pathology } \\
\hline LEP & 103 & 8.6 \\
\hline $\mathrm{ACN}$ & 558 & 46.7 \\
\hline PAP & 430 & 36 \\
\hline SOL & 52 & 4.3 \\
\hline MIP & 6 & 0.5 \\
\hline Others & 47 & 3.9 \\
\hline
\end{tabular}

Table 1 (continued)
Table 1 (continued)

\begin{tabular}{|c|c|c|}
\hline Variables & No. of patients & $(\%)$ \\
\hline \multicolumn{3}{|c|}{ Proposed new pathology } \\
\hline nLEP & 63 & 5.3 \\
\hline $\mathrm{nACN}$ & 276 & 23.1 \\
\hline nPAP & 648 & 54.2 \\
\hline nSOL & 137 & 11.4 \\
\hline nMIP & 36 & 3.0 \\
\hline Others & 36 & 3.0 \\
\hline \multicolumn{3}{|c|}{$8^{\text {th }}$ TNM classification } \\
\hline IA1 & 104 & 8.7 \\
\hline IA2 & 423 & 35.4 \\
\hline IA3 & 311 & 26 \\
\hline IB & 358 & 29.9 \\
\hline \multicolumn{3}{|l|}{ LN } \\
\hline$\leq 10$ & 803 & 67.1 \\
\hline$>10$ & 393 & 32.9 \\
\hline \multicolumn{3}{|l|}{$\mathrm{PI}$} \\
\hline No & 838 & 70.1 \\
\hline Yes & 358 & 29.9 \\
\hline \multicolumn{3}{|l|}{ LVI } \\
\hline No & 1,128 & 94.3 \\
\hline Yes & 68 & 5.7 \\
\hline
\end{tabular}

RUL, right upper lobe; RML, right middle lobe; $R L L$, right lower lobe; LUL, left upper lobe; LLL, left lower lobe; sublob, sublobectomy; lob, lobectomy; LEP, lepidic-predominant ADC; $A C N$, acinar-predominant ADC; PAP, papillary-predominant ADC; SOL, solid-predominant ADC; MIP, micropapillarypredominant ADC; nLEP, new classification of LEP; nACN, new classification of ACN; nPAP, new classification of PAP; nSOL, new classification of SOL; nMIP, new classification of MIP; TNM, tumor, node, metastasis; LN, harvested lymph nodes; PI, pleural invasion; LVI, lymphovascular invasion.

(8.7\%). For lymph nodes, we selected 10 as the cutoff value for the harvested lymph nodes, and patients with fewer than 10 lymph nodes still constituted the majority $(67.1 \%)$, with only $32.9 \%$ of patients having more than 10 harvested lymph nodes. Moreover, 29.9\% patients had pleural invasion (PI) in stage IB, indicating that stage IB 
Table 2 Univariable Cox proportional hazards regressions for patients with stage I invasive adenocarcinoma who underwent complete resection and lymph node dissection

\begin{tabular}{|c|c|c|c|c|c|c|}
\hline Variables & \multicolumn{3}{|c|}{ RFS } & \multicolumn{3}{|c|}{ os } \\
\hline Sex & 0.674 & $0.497-0.914$ & 0.011 & 0.545 & $0.352-0.843$ & 0.006 \\
\hline Age & 1.214 & $0.895-1.645$ & 0.213 & 1.166 & $0.754-1.803$ & 0.491 \\
\hline Smoking history & 1.375 & $1.012-1.869$ & 0.042 & 2.039 & $1.316-3.157$ & 0.001 \\
\hline Adjuvant chemotherapy & 1.888 & $1.370-2.601$ & $<0.001$ & 1.744 & $1.098-2.769$ & 0.018 \\
\hline Size & 1.561 & $1.210-2.014$ & 0.001 & 1.551 & $1.079-2.230$ & 0.018 \\
\hline Surgery & 0.883 & $0.391-1.995$ & 0.765 & 0.827 & $0.261-2.624$ & 0.748 \\
\hline Pathology & 1.058 & $0.913-1.227$ & 0.455 & 1.145 & $0.928-1.411$ & 0.206 \\
\hline LN & 2.337 & $0.851-2.998$ & 0.064 & 1.180 & $0.739-1.885$ & 0.488 \\
\hline $\mathrm{PI}$ & 2.024 & $1.490-2.748$ & $<0.001$ & 1.739 & $1.118-2.703$ & 0.014 \\
\hline LVI & 1.976 & $1.180-3.309$ & 0.010 & 1.961 & $0.945-4.071$ & 0.071 \\
\hline
\end{tabular}

RFS, recurrence-free survival; OS, overall survival; HR, hazard ratio; Cl, confidence interval; TNM, tumor, node, metastasis; LN, harvested lymph nodes; PI, pleural invasion; LVI, lymphovascular invasion.

or PI could be included in later multivariable analysis. Patients with lymphovascular invasion (LVI) accounted for only $5.7 \%$ of cases, which was likely related to the relatively restrained invasiveness of early-staged IADC in lymphatic vessels and surrounding blood vessels.

\section{Recurrence and survival analysis for pathological stage I IADC}

The median follow-up time was 51.51 months (range, 1-77 months), during which there were 166 (13.9\%) patients who relapsed (including local recurrence and distant metastasis) and $81(6.8 \%)$ patients who died (63 due to cancer-specific death and 18 deaths due to other causes). The 5 -year RFS and OS were $86.3 \%$ and $94.1 \%$, respectively (Figure S1).

\section{Univariable and multivariable cox regressions for pathological stage I IADC}

In the analysis of univariable cox regression, we enrolled all the related baseline clinicopathological data of resected stage I IADC patients into the Cox model and analyzed the potential risk factors that impacted the recurrence and survival outcome of pathological stage I IADC patients (Table 2). With respect to RFS, females demonstrated much better RFS compared with males (HR 0.674, 95\% CI, $0.497-0.914, \mathrm{P}=0.011)$, which indicated female sex as the potential protective factor of recurrence to pathological stage I IADC. As for smoking history, patients with a smoking history demonstrated worse recurrence as compared to never-smokers (HR 1.375, 95\% CI, 1.012 $1.869, \mathrm{P}=0.042$ ), and thus smoking history was indicated to be potential risk factor for postoperative recurrence. Patients who received adjuvant chemotherapy also demonstrated a worse recurrence rate compared to patients who did not receive adjuvant chemotherapy (HR 1.888, 95\% CI, 1.370-2.601, $\mathrm{P}<0.001$ ), a finding which requires further investigation. Tumor size as well as the eighth TNM classification was also associated with RFS rate (tumor size: HR 1.561, 95\% CI, 1.210-2.014, $\mathrm{P}=0.001$; TNM: HR 1.576, 95\% CI, 1.333-1.862, $\mathrm{P}<0.001)$. Furthermore, $\mathrm{PI}$ and LVI also showed themselves as potential recurrence factors for resected stage I IADC (PI: HR 2.024, 95\% CI, 1.490-2.748, $\mathrm{P}<0.001$; LVI: HR 1.976, $95 \%$ CI, $1.180-3.309, \mathrm{P}=0.010)$. In contrast to the traditional 
predominant subtypes, our proposed new classification of pathological stage I IADC was significantly associated with the recurrence outcome (HR 1.393, 95\% CI, 1.211-1.603, $\mathrm{P}<0.001)$, while predominant subtypes were not $(\mathrm{P}=0.455)$. Furthermore, patient age $(\mathrm{P}=0.213)$, tumor location $(\mathrm{P}=0.306)$, surgical approach $(\mathrm{P}=0.765)$, and harvested lymph nodes $(\mathrm{P}=0.064)$ also showed no statistical difference, indicating that they have no impact on the recurrence for pathological stage I IADC.

As for the clinical survival outcomes, female sex was still a potential protective factor for pathological stage I IADC (HR 0.545, 95\% CI, 0.352-0.843, P=0.006), while smoking history was a potential risk factor for OS (HR 2.039, 95\% CI, 1.316-3.157, P=0.001). However, those patients receiving postoperative chemotherapy still demonstrated potentially worse OS (HR 2.039, 95\% CI, $1.316-3.157, \mathrm{P}=0.001)$. Tumor size as well as the eighth TNM classification was also potential risk factors for OS (tumor size: HR 1.551, 95\% CI, 1.079-2.230, $\mathrm{P}=0.018$; TNM classification: HR 1.483, 95\% CI, 1.172-1.876, $\mathrm{P}=0.001)$. Furthermore, PI was also revealed to be a potential risk factor for OS (HR 1.739, 95\% CI, 1.118 $2.703, \mathrm{P}=0.014)$. In the univariable analysis of OS, our proposed new classification of pathological stage I IADC strongly indicated the potential ability for OS prognosis, with the similar results to RFS (HR 1.298, 95\% CI, 1.043-1.614, $\mathrm{P}=0.019)$. Meanwhile, age $(\mathrm{P}=0.491)$, tumor location $(\mathrm{P}=0.833)$, surgical approach $(\mathrm{P}=0.748)$, traditional predominant subtypes $(\mathrm{P}=0.206)$, harvested lymph node $(\mathrm{P}=0.488)$, and LVI $(\mathrm{P}=0.071)$ showed no statistical difference, indicating these have no prognostic ability in OS for resected stage I IADC.

After univariable analysis, we enrolled all the potential risk factors related to RFS and OS into the multivariable cox regression models, respectively (Table 3). Two independent risk factors were identified as being associated with cancer recurrence in our proposed pathological classification and TNM classification: nSOL (HR 5.428, 95\% CI, 1.277-23.074, P=0.022), nMIP (HR 10.784, 95\% CI, 2.415-48.154, $\mathrm{P}=0.002$ ), stage IA3 (HR 3.231, 95\% CI, 1.144-9.127, P=0.027), and stage IB (HR 4.202, 95\% CI, 1.504-11.736, $\mathrm{P}=0.006)$. Interestingly, the predominant patterns were not associated with postsurgical relapse.

With respect to OS, our proposed pathological classification, similar to the conventional predominant patterns, did not show any statistical influence, while stage IB and smoking history strongly corresponded with worse survival outcome (Tables 2,3).

\section{The RFS-related risk stratification model for pathological stage I IADC}

Accordingly, the results of RFS multivariable analysis were applied and $\log -\mathrm{HR}$ was calculated and standardized, from which the personalized scores were derived in turn (Table 4). The highest scores were 2 points, and the lowest one was 0 . Among all the independent recurrent risk factors of resected stage I IADC, nMIP received the most points ( 2 points), followed by nSOL (1.4 points), stage IB (1.2 points), and stage IA3 (1 point), with nLEP, nACN, nPAP, etc., as well as stage IA1 and IA2, all receiving 0 points.

The risk stratification model based on our proposed new pathological classification and the eighth TNM classification was established according to the statistical analysis above (Figure $2 A$ ), and the corresponding 5 -year RFS of low-risk group, intermediate-risk group and highrisk group in the risk stratification model was $93.0 \%, 81.6 \%$ and $64.4 \%$, respectively. Furthermore, the survival curve of the eighth TNM classification was also plotted (Figure 2B), indicating a 5 -year RFS of stage IA1, stage IA2, stage IA3 and stage IB was $96.0 \%, 89.4 \%, 83.1 \%$ and $76.1 \%$, respectively.

In order to compare the prognostic capability between those 2 above approaches, ROCs were plotted by means of AUC with the SPSS software. After these 2 approaches were compared in terms of AUCs of different postoperative times (1-, 3-, 5-year, and total postoperative time), we found that there was a difference in recurrence prediction between the risk stratification model and the eight TNM classification across the different postoperative times.

For 1-year recurrence prediction of resected stage I IADC, the risk stratification model demonstrated superiority, as it could much more precisely predict the prognosis of 1-year recurrence after surgery (1-year: model vs. $\mathrm{TNM}=0.653$ vs. $0.556 ; \mathrm{P}=0.033)$.

For 3-year prediction, our risk stratification model still showed better performance compared to the traditional TNM classification (3-year: model vs. $\mathrm{TNM}=0.663 v s$. 0.607; $\mathrm{P}=0.008$ ). All the ROC curves and AUCs for these performances are shown in Figure 3A,B, respectively.

For 5-year and total RFS prediction, however, no remarkable difference in prognostic prediction was found, which indicated that our risk stratification model had no prognostic advantage for long-term recurrence compared 
Table 3 Multivariable Cox proportional hazards regressions for patients with stage I invasive adenocarcinoma who underwent complete resection and lymph node dissection

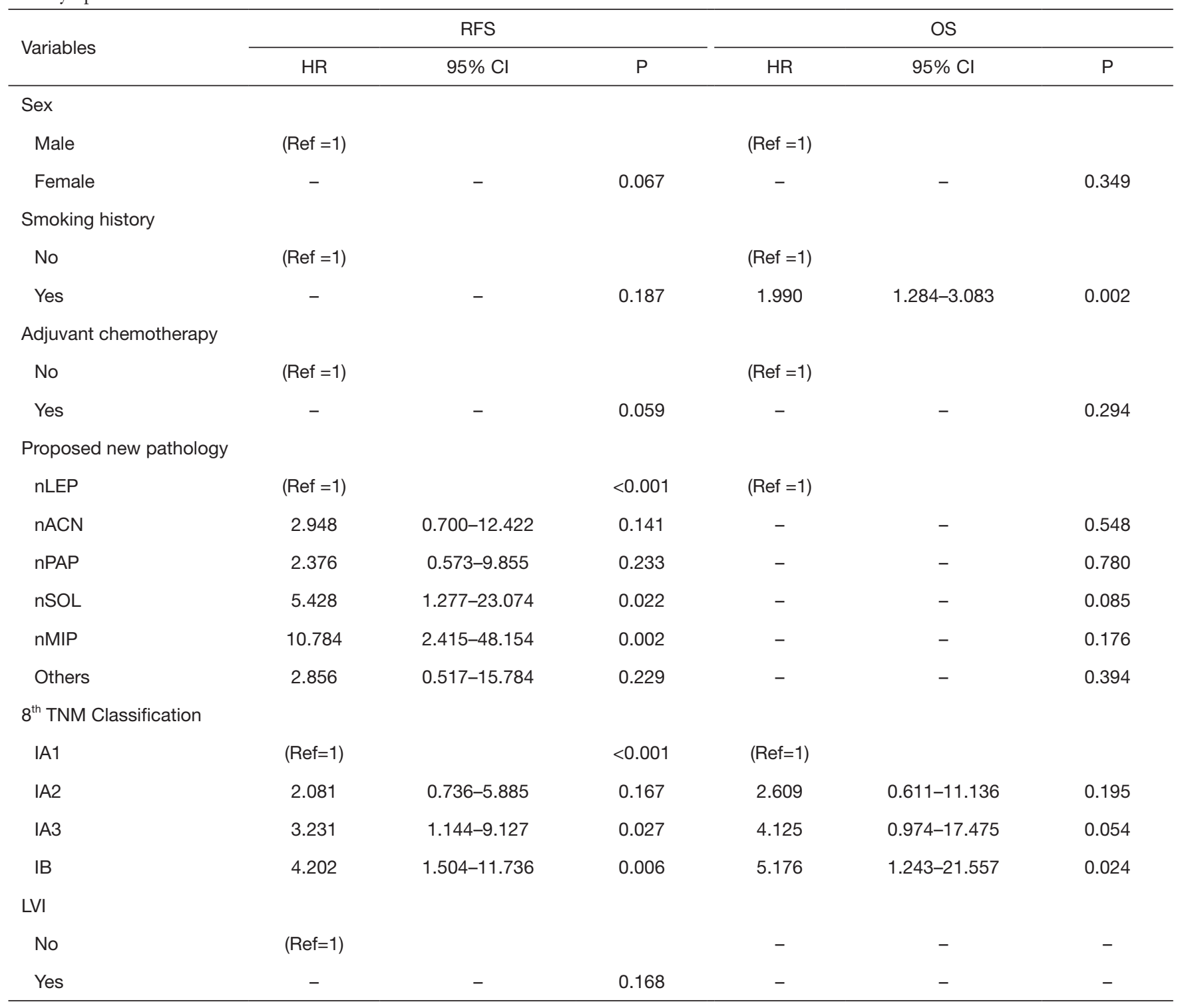

RFS, recurrence-free survival; OS, overall survival; Ref, reference; HR, hazard ratio; $\mathrm{Cl}$, confidence interval; nLEP, new classification of LEP; nACN, new classification of ACN; nPAP, new classification of PAP; nSOL, new classification of SOL; nMIP, new classification of MIP; TNM, tumor, node, metastasis; PI, pleural invasion; LVI, lymphovascular invasion.

Table 4 Standardized risk score coefficients based on recurrence-free survival

\begin{tabular}{|c|c|c|c|c|c|}
\hline Recurrence-free Survival & \multicolumn{5}{|c|}{ Scores } \\
\hline Proposed new pathology & nLEP/nACN/nPAP/Others & & & $\mathrm{nSOL}$ & nMIP \\
\hline $8^{\text {th }}$ TNM classification & $\mathrm{IA} 1$ and IA2 & IA3 & IB & & \\
\hline
\end{tabular}


A

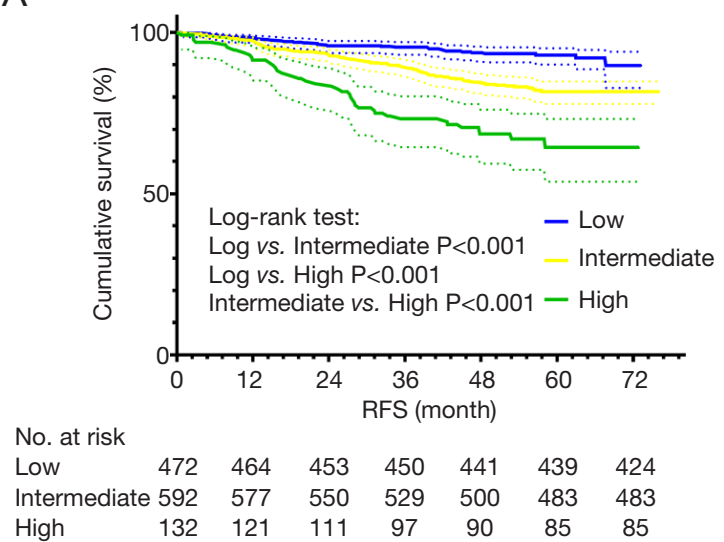

B

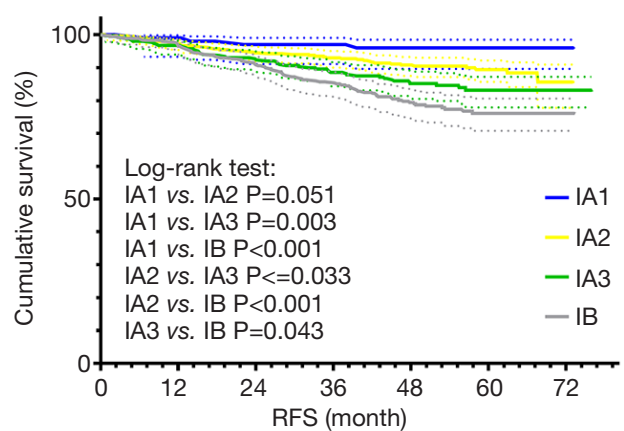

No. at risk

$\begin{array}{llllllll}\text { IA1 } & 104 & 103 & 101 & 101 & 100 & 100 & 100 \\ \text { IA2 } & 423 & 416 & 400 & 393 & 384 & 378 & 362 \\ \text { IA3 } & 311 & 301 & 288 & 275 & 265 & 258 & 258 \\ \text { IB } & 358 & 347 & 326 & 307 & 284 & 272 & 272\end{array}$

Figure 2 Recurrence-free survival according to the risk stratification model (A) and the eighth TNM classification (B).

A

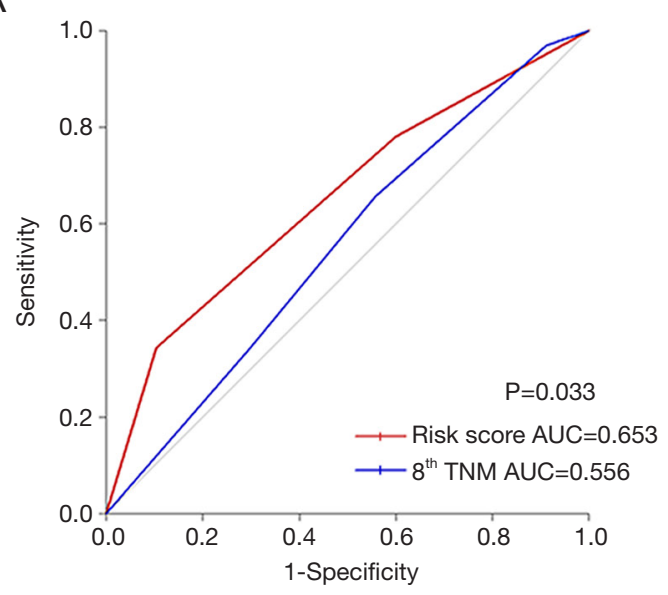

C

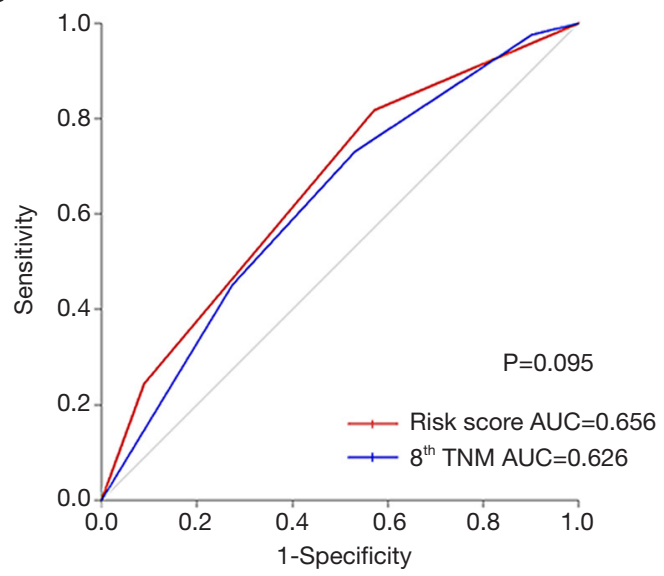

B

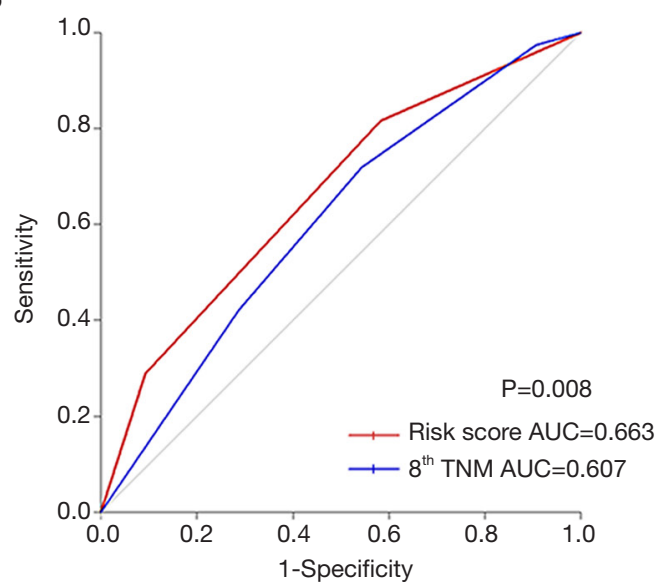

D

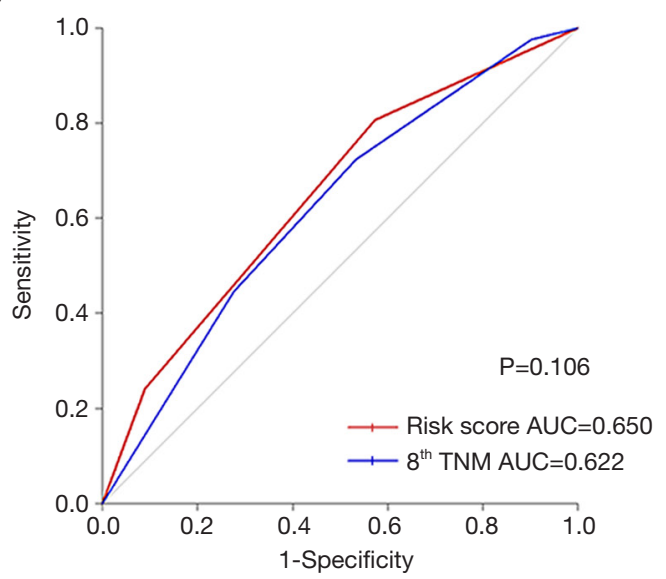

Figure 3 Prognostic performance comparison between risk stratification model and the eighth TNM classification for recurrence-free survival. AUC comparison between the risk model and TNM classification in 1-year RFS (A), 3-year RFS (B), 5-year RFS (C), and total RFS (D). 
with TNM classification (5-year: model $v s$. TNM $=0.656$ vs. $0.626, \mathrm{P}=0.095$; total: model vs. $\mathrm{TNM}=0.650$ vs. 0.622 , $\mathrm{P}=0.106)$. The ROC curves and the related AUCs for these performances were shown in Figure 3C,D, respectively.

\section{The OS-related risk stratification model for pathological stage I IADC}

As for the risk model of OS, individualized scores were also established in accordance with previous methods (Table S2). Among all the independent risk scores for OS, the highest score was 2.4 points, and the lowest one score was 0 points. Among all the independent risk factors, stage IB had 2.4 points, followed by smoking history with 1 point. Meanwhile, never-smokers, stage IA1, stage IA2, and stage IA3, all received 0 points.

We thus established the risk stratification model for OS prediction using different risk scores of different patients; the cutoffs for the low-risk, intermediate-risk, and highrisk group are mentioned in the Methods. Furthermore, the stratified survival curves of the risk model and TNM system are presented in Figure S2.

To compare the sensitivity and specificity of OS prediction between the risk stratification model and the eighth TNM classification, we created ROC curves and AUCs using SPSS software. However, in contrast to the results mentioned above, the risk model did not demonstrate significant predictive ability in short-term survival prognosis compared with TNM system, meaning our risk stratification model was not superior to the traditional TNM classification for short-term postoperative survival (1-year: model vs. $\mathrm{TNM}=0.396$ vs. $0.431, \mathrm{P}=0.822 ; 3$-year: model vs. $\mathrm{TNM}=0.608$ vs. $0.592, \mathrm{P}=0.648$ ). Additionally, the risk stratification model and TNM classification system showed no difference in prognostic ability for the long-term survival outcome of resected stage I IADC (5-year: model vs. $\mathrm{TNM}=0.599$ vs. $0.594, \mathrm{P}=0.836$; total: model $v s$. TNM $=0.610$ vs. $0.602, \mathrm{P}=0.740$ ). All the related ROC curves and AUCs are plotted in the Figure S3A,B,C,D.

\section{Discussion}

With the discovery of the activation of epidermal growth factor receptor (EGFR) mutations in 2004, it was expected that the application of lung cancer gene-specific diagnosis and therapy would bring about a revolution in the clinical management of lung cancer (19). In addition to EGFR, several genes including $A L K$ and ROS1, along with their related targeted therapy were also confirmed as representing promising treatment options for advanced and stage IV NSCLC $(20,21)$. Eventually, there were more protocols and clinical practice management guidelines created for the suggested targeted treatments $(22,23)$. As genes for as routine practice among resected early-staged ADC have not been tested and their prognostic significance is still not confirmed, it is still of great importance for clinicians to evaluate and predict precise recurrence and survival outcome for early-staged ADC patients using the clinical and pathological characteristics.

According to the novel ADC pathological classification published by the IASLC/ATS/ERS in 2011, any histological pattern should be recorded with an assessment quantified by $5 \%$ increments with a single predominant pattern being recommended to represent the certain tumor (11). While approximately $8-90 \%$ of IADCs have been recognized to be the mixed patterns, focusing on a specific predominant pattern while ignoring the coexisting ones might lead to incorrect prognostic assessment (12). To date, large numbers of previous studies have proven that SOL/MIP are associated with worse clinical outcome $(24,25)$; MIP is particularly correlated with a higher degree of aggressiveness, LVI, and lymph node metastasis (26,27). Interestingly, MIP still show a consistently prognostic impact, even when the presence is $\geq 5 \%$ or $\geq 1 \%$ $(12,14,16,28,29)$. Thus, it was reasonable to speculate that an MIP component could become a representative classification of certain ADC types even when not predominant. Compared with MIP, SOL displayed limited prognostic impact when not coexistent with MIP $(30,31)$. Therefore, the reclassification of nSOL was proposed. Previous studies have uniformly classified ACN/PAP into the same group for the relatively similar prognostic outcome (12). Nevertheless, we tried to separate these 2 patterns in our proposed classification. Interestingly, in contrast to the eighth TNM classification system, our proposed system demonstrated marked superiority in early-stage ADC recurrence prediction. From this we could recognize that harboring MIP could definitively determine the prevalence of disease relapse regardless of whether a predominant pattern was present. This "all or none" effect was also present in nSOL, indicating that the higher risk of recurrence was mostly an influence of the SOL component. Therefore, the classification of IADC could be reclassified according to certain type of pathology instead of predominant one. Conversely, nPAP/nACN showed no statistical difference in RFS or OS, probably 
due to the prevalence of LEP shows a tendency of better prognosis (14), thus prompting for further investigation of the latter's prognostic effect.

Pignon et al. and Chansky et al. have pointed out that almost $50 \%$ of early-staged NSCLC suffer from cancer relapse $(32,33)$. Although, the current TNM system has, for decades, maintained its significance in static and universal applicability, its prognostic ability has been constantly scrutinized and modified $(9,34)$. We noticed that this conventional staging system was becoming less useful, especially among early-staged ADC when the T category became the only variable for prognosis analysis. In this research, our risk stratification model for RFS was constructed with the combination of two well-known and easy-to-extract clinicopathological system, our proposed pathological classification and the eighth TNM system, all of which were verified and accepted through multivariable Cox regressions. After being stratified into 3 risk levels by personalized scores, the risk model showed higher prognostic sensitivity and specificity compared with the traditional TNM classification in early recurrence prediction. Furthermore, the high-risk group could also be a better indicator for adjuvant therapy, owing to the MIP and SOL were contained in this group which was considered as a high risk for cancer recurrence $(12,30)$. A clear statistical difference was evident between our risk model and TNM classification for 5-year and long-term recurrence prediction, implying an indispensable role of the traditional TNM classification in long-term prognosis of ADC recurrence. As for OS, our proposed pathological classification did not perform significantly better, strongly suggesting relatively low importance of histological subtypes on clinical survival outcome, which was consistent with a previous study (12). With consideration to these findings, the TNM classification can still be said to be unparalleled in its ability in predicting survival prognosis. Furthermore, smoking history also indicated poor OS, as previously suggested (35). Unfortunately, the risk model based on these 2 independent factors did not demonstrate significant superiority over conventional TNM classification in predicting OS.

In addition to this risk stratification model for early recurrence prediction, several risk factors, including tumor budding, tumor spread through air spaces (STAS), and even liquid biopsy, have been recently verified as reliable independent predictors of postoperative recurrence for resected $\mathrm{ADC}$ patients $(36,37)$. It is therefore necessary to reconsider and recombine these potential risk factors into our risk stratification model for future work.

There were several limitations in this research. First, this study retrospectively collected patients from 2 hospitals. The nature of this retrospective analysis inevitably introduced biases, in that our sample population likely lacks universality and representativeness. Second, although HE slides were all double-checked by 2 experienced pathologists in each hospital, the subjective nature of this examination might have led to discrepancies between pathologists. Third, we determined that any pathological pattern should be recorded when it showed $\geq 5 \%$ predominance. For example, nMIP was noted when MIP was $\geq 5 \%$. On the other hand, we did not consider the component when it was $<5 \%$. The consequences of using this cutoff are unknown and require further investigation.

\section{Conclusions}

Harboring a certain pattern of a histological subtype, such as MIP/SOL, instead of merely considering the predominant subtype, could be recommended as a new kind of representative classification, and this classification was clearly correlated with worse RFS. Furthermore, the risk stratification model based on this proposed pathological classification showed clear superiority for predicting early recurrence of resected stage I ADC when compared to the eighth TNM classification, suggesting that the influence of certain pathological subtypes should not be neglected when estimating potential recurrence risk.

\section{Acknowledgments}

The authors appreciate the academic support from AME Lung Cancer Collaborative Group.

Funding: This work was supported by the National Natural Science Foundation of China (no. 81572253 and 81372525).

\section{Footnote}

Reporting Checklist: The authors have completed the TRIPOD reporting checklist. Available at http://dx.doi. org/10.21037/tlcr-21-393

Data Sharing Statement: Available at http://dx.doi. org/10.21037/tlcr-21-393

Conflicts of Interest: All authors have completed the ICMJE uniform disclosure form (available at http://dx.doi. 
org/10.21037/tlcr-21-393). The authors have no conflicts of interest to declare.

Ethical Statement: The authors are accountable for all aspects of the work in ensuring that questions related to the accuracy or integrity of any part of the work are appropriately investigated and resolved. All procedures performed in this study involving human participants were in accordance with the Declaration of Helsinki (as revised in 2013). The study was approved by ethics board of Shanghai Chest Hospital (KS1992) and informed consent was not needed.

Open Access Statement: This is an Open Access article distributed in accordance with the Creative Commons Attribution-NonCommercial-NoDerivs 4.0 International License (CC BY-NC-ND 4.0), which permits the noncommercial replication and distribution of the article with the strict proviso that no changes or edits are made and the original work is properly cited (including links to both the formal publication through the relevant DOI and the license). See: https://creativecommons.org/licenses/by-nc-nd/4.0/.

\section{References}

1. Siegel R, Naishadham D, Jemal A. Cancer statistics, 2013. CA: Cancer J Clin 2013;63:11-30.

2. Chen $\mathrm{W}$, Zheng R, Baade PD, et al. Cancer statistics in China, 2015. CA Cancer J Clin 2016;66:115-32.

3. Travis WD. Pathology of lung cancer. Clin Chest Med 2011;32:669-92.

4. Ginsberg RJ, Rubinstein LV. Randomized trial of lobectomy versus limited resection for T1 N0 non- small cell lung cancer. Lung Cancer Study Group. Ann Thorac Surg 1995;60:615-622, discussion 622-613.

5. Landreneau RJ, Normolle DP, Christie NA, et al. Recurrence and Survival Outcomes After Anatomic Segmentectomy Versus Lobectomy for Clinical Stage I Non-Small-Cell Lung Cancer: A Propensity-Matched Analysis. J Clin Oncol 2014;32:2449-55.

6. Dai C, Shen J, Ren Y, et al. Choice of Surgical Procedure for Patients With NSCLC $\leq 1 \mathrm{~cm}$ or 1 to $2 \mathrm{~cm}$ Among Lobectomy, Segmentectomy, and Wedge Resection: A Population-Based Study. J Clin Oncol 2016;34:3175-82.

7. Hung JJ, Jeng WJ, Hsu WH, et al. Predictors of death, local recurrence, and distant metastasis in completely resected pathological stage-I non-small-cell lung cancer. J Thorac Oncol 2012;7:1115-23.
8. Christensen JD, Chiles C. Low-Dose Computed Tomographic Screening for Lung Cancer. Clin Chest Med 2015;36:147-60, vii.

9. Detterbeck FC, Boffa DJ, Kim AW, et al. The Eighth Edition Lung Cancer Stage Classification. Chest 2017;151:193-203.

10. Travis WD, Brambilla E, Nicholson AG, et al. The 2015 World Health Organization classification of lung tumors: impact of genetic, clinical and radiologic advances since the 2004 classification. J Thorac Oncol 2015;10:1243-60.

11. Travis WD, Brambilla E, Noguchi M, et al. International Association for the Study of Lung Cancer/American Thoracic Society/European Respiratory Society international multidisciplinary classification of lung adenocarcinoma. J Thorac Oncol 2011;6:244-85.

12. Tsao MS, Marguet S, Le Teuff G, et al. Subtype Classification of Lung Adenocarcinoma Predicts Benefit From Adjuvant Chemotherapy in Patients Undergoing Complete Resection. J Clin Oncol 2015;33:3439-46.

13. Yoshiya T, Mimae T, Tsutani Y, et al. Prognostic Role of Subtype Classification in Small-Sized Pathologic N0 Invasive Lung Adenocarcinoma. Ann Thorac Surg 2016;102:1668-73.

14. Yanagawa N, Shiono S, Abiko M, et al. The Clinical Impact of Solid and Micropapillary Patterns in Resected Lung Adenocarcinoma. J Thorac Oncol 2016;11:1976-83.

15. Liu S, Wang R, Zhang Y, et al. Precise Diagnosis of Intraoperative Frozen Section Is an Effective Method to Guide Resection Strategy for Peripheral Small-Sized Lung Adenocarcinoma. J Clin Oncol 2016;34:307-13.

16. Zhao Y, Wang R, Shen X, et al. Minor Components of Micropapillary and Solid Subtypes in Lung Adenocarcinoma are Predictors of Lymph Node Metastasis and Poor Prognosis. Ann Surg Oncol 2016;23:2099-105.

17. Rassi A Jr, Rassi A, Little WC, et al. Development and validation of a risk score for predicting death in Chagas' heart disease. N Engl J Med 2006;355:799-808.

18. Pilotto S, Sperduti I, Novello S, et al. Risk Stratification Model for Resected Squamous-Cell Lung Cancer Patients According to Clinical and Pathological Factors. J Thorac Oncol 2015;10:1341-8.

19. Lynch TJ, Bell DW, Sordella R, et al. Activating mutations in the epidermal growth factor receptor underlying responsiveness of non-small-cell lung cancer to gefitinib. N Engl J Med 2004;350:2129-39.

20. Park K, Tan EH, O'Byrne K, et al. Afatinib versus gefitinib as first-line treatment of patients with EGFR mutationpositive non-small-cell lung cancer (LUX-Lung 7): a phase 
2B, open-label, randomized controlled trial. Lancet Oncol 2016;17:577-89.

21. Goss G, Tsai CM, Shepherd FA, et al. Osimertinib for pretreated EGFR Thr790Met-positive advanced nonsmall-cell lung cancer (AURA2): a multicentre, open-label, single-arm, phase 2 study. Lancet Oncol 2016;17:1643-52.

22. Masters GA, Temin S, Azzoli CG, et al. Systemic Therapy for Stage IV Non- Small-Cell Lung Cancer: American Society of Clinical Oncology Clinical Practice Guideline Update. J Clin Oncol 2015;33:3488-515.

23. Tan DS, Yom SS, Tsao MS, et al. The International Association for the Study of Lung Cancer consensus statement on optimizing management of EGFR mutation positive non-small cell lung cancer: status in 2016. J Thorac Oncol 2016;11:946-63.

24. Russell PA, Wainer Z, Wright GM, et al. Does lung adenocarcinoma subtype predict patient survival? A clinicopathologic study based on the new International Association for the Study of Lung Cancer/American Thoracic Society/European Respiratory Society international multidisciplinary lung adenocarcinoma classification. J Thorac Oncol 2011;6:1496-504.

25. Ujiie H, Kadota K, Chaft JE, et al. Solid predominant histologic subtype in resected stage I lung adenocarcinoma is an independent predictor of early, extrathoracic, multisite recurrence and of poor postrecurrence survival. J Clin Oncol 2015;33:2877-84.

26. Tsubokawa N, Mimae T, Sasada S, et al. Negative prognostic influence of micropapillary pattern in stage IA lung adenocarcinoma. Eur J Cardiothorac Surg 2016;49:293-9.

27. Yoshida Y, Nitadori JI, Shinozaki-Ushiku A, et al. Micropapillary histological subtype in lung adenocarcinoma of $2 \mathrm{~cm}$ or less: impact on recurrence and clinical predictors. Gen Thorac Cardiovasc Surg 2017;65:273-9.

28. Nitadori J, Bograd AJ, Kadota K, et al: Impact of

Cite this article as: Wang Y, Zheng D, Luo J, Zhang J, Pompili C, Ujiie H, Matsuura N, Chen H, Yao F. Risk stratification model for patients with stage I invasive lung adenocarcinoma based on clinical and pathological predictors. Transl Lung Cancer Res 2021;10(5):2205-2217. doi: 10.21037/ tlcr-21-393 micropapillary histologic subtype in selecting limited resection vs lobectomy for lung adenocarcinoma of $2 \mathrm{~cm}$ or smaller. J Natl Cancer Inst 2013;105:1212-20.

29. Maeda R, Isowa N, Onuma H, et al. Lung adenocarcinomas with micropapillary components. Gen Thorac Cardiovasc Surg 2009;57:534-9.

30. Warth A, Muley T, Meister M, et al. The novel histologic International Association for the Study of Lung Cancer/ American Thoracic Society/European Respiratory Society classification system of lung adenocarcinoma is a stage-independent predictor of survival. J Clin Oncol 2012;30:1438-46.

31. Cha MJ, Lee HY, Lee KS, et al. Micropapillary and solid subtypes of invasive lung adenocarcinoma: clinical predictors of histopathology and outcome. J Thorac Cardiovasc Surg 2014;147:921-8.e2.

32. Pignon JP, Tribodet H, Scagliotti GV, et al. Lung adjuvant cisplatin evaluation: A pooled analysis by the LACE Collaborative Group. J Clin Oncol 2008;26:3552-9.

33. Chansky K, Sculier JP, Crowley JJ, et al. The International Association for the Study of Lung Cancer Staging Project: Prognostic factors and pathologic TNM stage in surgically managed non-small cell lung cancer. J Thorac Oncol 2009;4:792-801.

34. Detterbeck F. Stage classification and prediction of prognosis: The difference between accountants and speculators. J Thorac Oncol 2013;8:820-2.

35. Eng L, Su J, Qiu X, et al. Second-Hand Smoke As a Predictor of Smoking Cessation Among Lung Cancer Survivors. J Clin Oncol 2014;32:564-70.

36. Abbosh C, Birkbak NJ, Wilson GA, et al. Phylogenetic ctDNA analysis depicts early stage lung cancer evolution. Nature 2017;545:446-51.

37. Jamal-Hanjani M, Wilson GA, McGranahan N, et al. Tracking the Evolution of Non-Small-Cell Lung Cancer. N Engl J Med 2017;376:2109-21. 


\section{Supplementary}

Table S1 The relationship between the predominant patterns of adenocarcinoma and the proposed new pathological classification of IADC.

\begin{tabular}{|c|c|c|c|c|c|c|c|}
\hline New pathology & LEP & ACN & PAP & SOL & MIP & Others & Total \\
\hline nLEP & 61 & 0 & 0 & 0 & 0 & 2 & 63 \\
\hline$n A C N$ & 25 & 247 & 0 & 0 & 0 & 4 & 276 \\
\hline nPAP & 15 & 233 & 396 & 0 & 0 & 4 & 648 \\
\hline nSOL & 2 & 66 & 17 & 52 & 0 & 0 & 137 \\
\hline nMIP & 0 & 12 & 17 & 0 & 6 & 1 & 36 \\
\hline Others & 0 & 0 & 0 & 0 & 0 & 36 & 36 \\
\hline Total & 103 & 558 & 430 & 52 & 6 & 47 & 1196 \\
\hline
\end{tabular}

Abbreviations: LEP, lepidic-predominant ADC; ACN, acinar-predominant ADC; PAP, papillary-predominant ADC; SOL, solid-predominant ADC; MIP, micropapillary-predominant ADC; nLEP, new classification of LEP; nACN, new classification of ACN; nPAP, new classification of PAP; nSOL, new classification of SOL; nMIP, new classification of MIP.
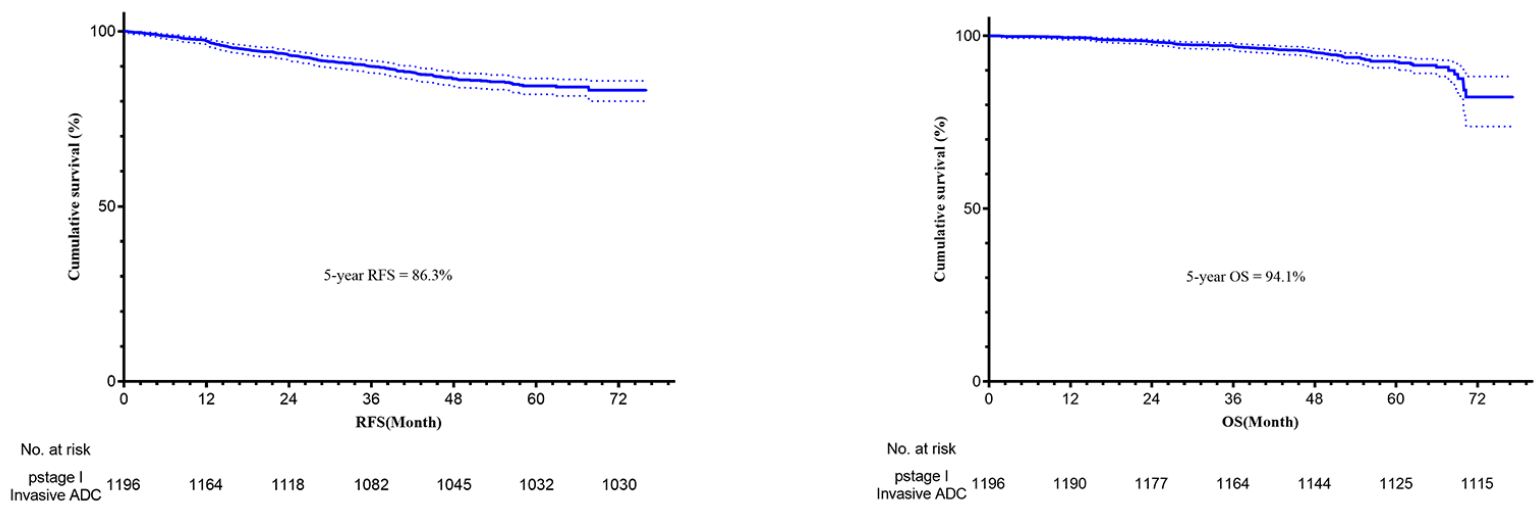

Figure S1 Recurrence-free survival and overall survival in the primary cohort. Kaplan-Meier curve for relapse-free survival and overall survival in 1,196 patients with resected stage I IADC.

Table S2 Standardized risk score coefficients based on overall survival.

\begin{tabular}{lccc}
\hline \multirow{2}{*}{ Overall survival } & & Scores & 2.4 \\
\cline { 2 - 4 } & 0 & 1 & Yes \\
\hline Smoking history & No & & IB \\
$8^{\text {th }}$ TNM classification & IA1/IA2/IA3 & & \\
\hline
\end{tabular}

Abbreviations: TNM, tumor, node, metastasis. 
A

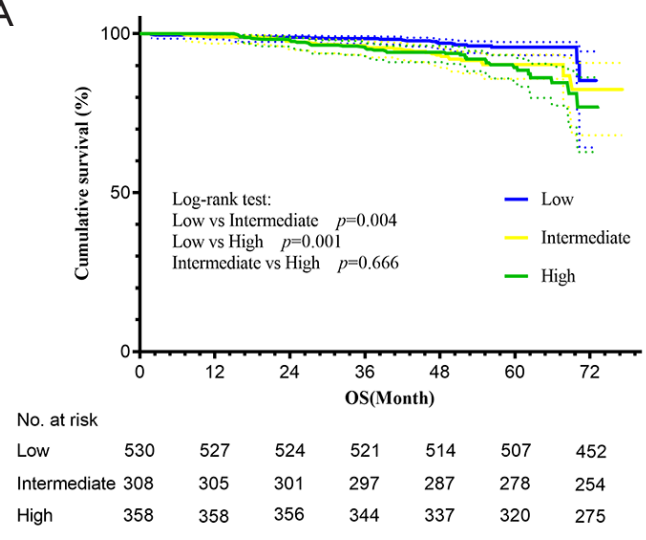

B

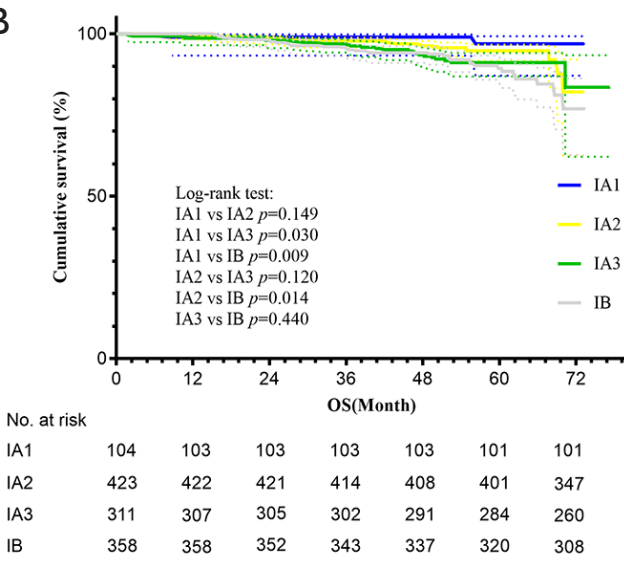

Figure S2 Overall survival according to the risk stratification model (A) and the eighth TNM classification (B). Kaplan-Meier curves for overall survival in 1,196 patients with resected stage I IADC, plotted by risk stratification model and the eighth TNM Classification, respectively.

A

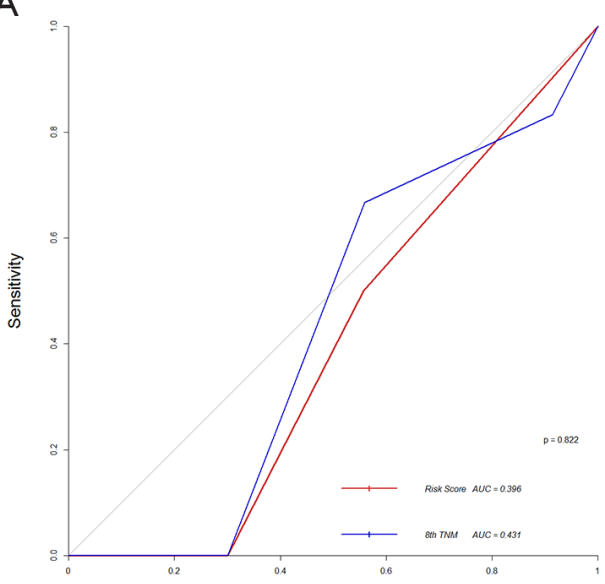

$\mathrm{C}$

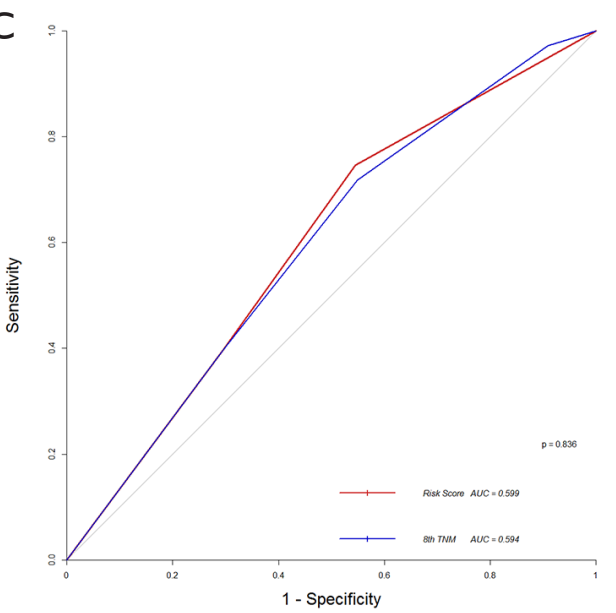

B

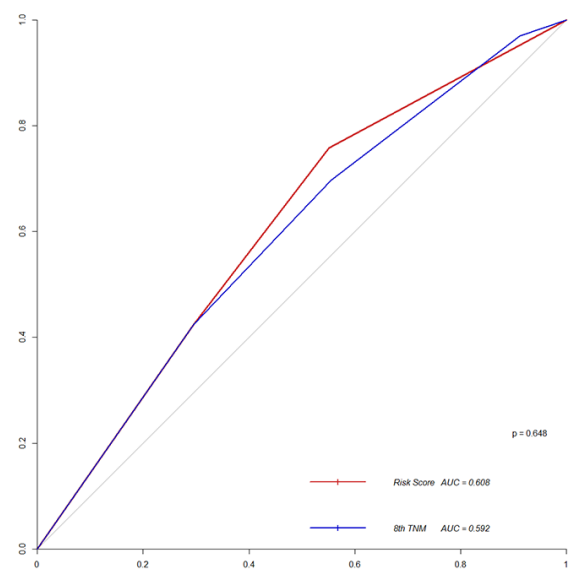

$\mathrm{D}$

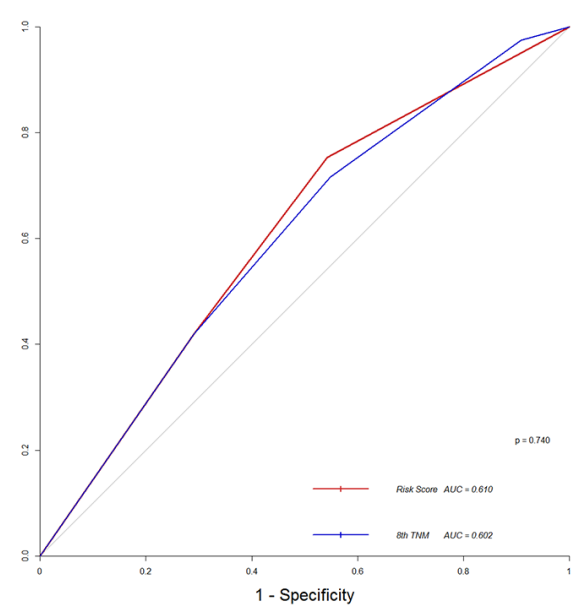

Figure S3 Prognostic performance comparison between risk stratification model and the eighth TNM classification for overall survival. AUC comparison between the risk model and TNM classification in 1-year OS (A), 3-year OS (B), 5-year OS (C), and total OS (D). 